\title{
An Analysis of Achievable Highest SLL in Moderate Phased Arrays with Quantized Control
}

\author{
Ajay Kochar \\ Electrical Communication Engineering Dept. \\ Indian Institute of Science \\ Bangalore, India \\ ajaykochar@iisc.ac.in
}

\author{
K. J. Vinoy \\ Electrical Communication Engineering Dept. \\ Indian Institute of Science \\ Bangalore, India \\ kjvinoy@iisc.ac.in
}

\begin{abstract}
Phased arrays with discrete control circuitry for amplitudes and phases are analysed to investigate the impact of finite resolution phase sifters and $R F$ attenuators on the achievable highest side lobe level (HSLL). Finite dynamic range offered by RF attenuators also limits the value of HSLL that can be achieved. The achieved HSLL may lie between 25 to $40 \mathrm{~dB}$ for practical values of dynamic range, depending on the array size and number of control bits. This aspect is studied for Chebyshev and discrete prolate spheroidal sequences (DPSS) arrays of different lengths. Finally, a novel approach for quantizing phases is proposed for reducing HSLL degradation.

Index Terms-Phased arrays, quantization, attenuators, phase shifters, Chebyshev, DPSS
\end{abstract}

\section{INTRODUCTION}

Analog beam forming and beam steering using phased arrays have been found to be effective in RADARS, wireless communication, and imaging for medical and other applications. Although digital baseband beamforming has been demonstrated in many of these cases, analog or hybrid approaches are likely to prevail for $5 \mathrm{G} \mathrm{mm}$ wave beamforming arrays [1]. Analog steering using digital controls provides better coverage at lower cost than full digital baseband beamforming approaches. However, practical constraints of these digital control circuitries may potentially limit their use in phased arrays.

The control circuits in analog phased arrays at $\mathrm{mm}$ wave consists of phase shifters and attenuators, both of which are known to have a finite resolution [2]. The number of bits used in the control circuitry is determined by budget and size constraints. Increasing the number of bits also increases power consumption. Thus, techniques to compensate for the impairments caused in the array pattern by low resolution phase shifters (and attenuators) are required. In addition, directive pattern of the antenna element may also distort the side lobes.

Most research work so far address the mean value of parasitic SLL [3], [4], [5] due to quantization in arrays with isotropic elements. Further, this mean parasitic SLL effect is analysed usually for large arrays, based on an approximation wherein the summation in the array factors are converted into integrals. Approaches to minimize errors are either based on randomization of quantized phases or by searching randomly over a part of a quantized grid. Consequently, the number of iterations is large and the improvement in HSLL is minor [6]. A comparison and mathematical treatment of various classical phase randomization techniques is provided in [3]. A simple technique to obtain equal side lobe patterns for microstrip patch arrays was recently communicated in [7], [8].

In this paper we analyze the relationship between the achieved HSLL and the dynamic range, and the resolution of attenuators and phase shifters in moderately sized linear arrays of isotropic elements. This paper is organized as follows. Section II analyses the effect of aforementioned impairments on phased arrays. Section III proposes a new method for realizing the phases to reduce HSLL degradation in small/moderate arrays.

\section{Analysis of Phased Array Performance with REALISTIC FEED NETWORK}

Limitations of realistic components in the feed network affects the radiation pattern of the antenna array, including its main beam direction and HSLL. HSLL is often expressed in $\mathrm{dB}$ relative to the peak amplitude. The complex array factor of a uniform linear array (ULA) of $\mathrm{N}$ elements with a spacing of $d$, with its main beam scanned to $\phi_{o}$ is [9]

$$
A F\left(\phi, \phi_{o}\right)=\sum_{n=1}^{N} a_{n} e^{j n k d\left(\sin \phi-\sin \phi_{o}\right)}
$$

where $k=2 \pi / \lambda, \lambda$ denoting the wavelength. The element spacing $d$ is normally taken as $0.5 \lambda$, and the useful coverage of ULA is $\left\{\Omega_{\phi} \mid-\pi \leq \Omega_{\phi} \leq \pi\right\}$, where $\Omega_{\phi}$ is the normalised spatial frequency $\Omega_{\phi}=\pi \sin \phi$. The progressive phase shift $\psi_{n}=n \pi \sin \phi_{0}$ decides the direction of the main beam peak while amplitudes $a_{n}$ are used for controlling the beamwidth and side lobe level (SLL).

In this work, linear antenna arrays with Chebyshev and Discrete Prolate Slepian Sequences (DPSS) [10] weights have been analysed for different HSLL targets. DPSS weights corresponding to maximum eigenvalue are selected out of $N$ sequences obtained from the optimization problem maximizing the ratio of the energy in the main beam (between first nulls) to the total energy of the array factor [11], [12]. Both DPSS and Chebyshev approaches result in wide range of amplitudes at different elements of the array, depending on 


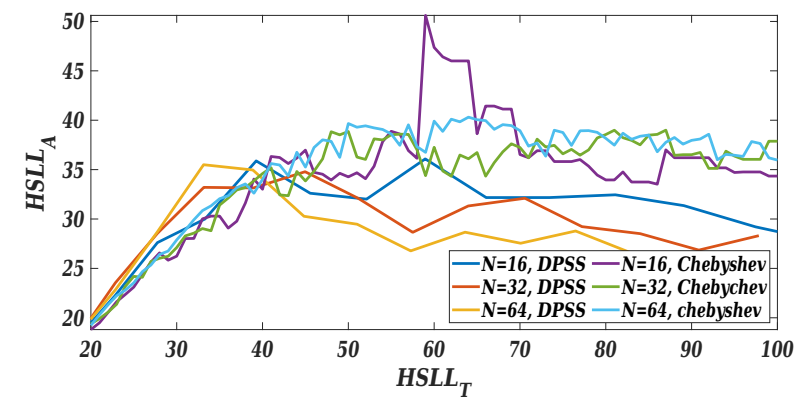

Fig. 1: Variation of $H S L L_{A}$ with $H S L L_{T}$ for chebyshev and DPSS tapers $\left(K_{A}=5\right.$ and $\left.D=31 d B\right)$.

its size and beamwidth/HSLL. In phased arrays fed with TR modules, these amplitudes are set using digitally controlled attenuators, which have a finite dynamic range $(D)$ and a limited number of bits $\left(K_{A}\right)$ for their control. Similarly, the precision of phase shifters $\left(K_{P}\right)$ is also finite. It has been found that the achieved HSLL, $H S L L_{A}$ is a function of $K_{P}$, $K_{A}$ and $D$, in addition to the number of elements, $N$. HSLL is calculated by first evaluating the AF to find its peaks, which are then sorted in decreasing order of their values. The second peak corresponds to $H S L L_{A}$, which is the worst case side lobe level.

\section{A. Effects of Practical Attenuators}

The effect of finite $D$ of attenuators is investigated first. Let $I=\left\{i_{n}\right\}_{n=1}^{N}$ denote the amplitude values (expressed in $\mathrm{dB}$, $\left.i_{n}=20 \log _{10} a_{n}\right)$. For an attenuator with $K_{A}$ bits, the step size is given by

$$
\Delta_{A}=D / 2^{K_{A}}
$$

The quantized value of $n t h$ amplitude is obtained by rounding:

$$
\hat{i}_{n}=\Delta_{A}\left[\frac{i_{n}}{\Delta_{A}}\right],\left|i_{n}\right| \leq D
$$

where $[*]$ denotes the simple rounding operation. However, due to the finite dynamic range,

$$
\hat{i}_{n}=-D, \text { if }\left|i_{n}\right| \geq D
$$

In this analysis, true (unquantized) values of phases were assumed at all elements. Further, for simplicity, the mainbeam is directed towards boresight. Therefore all phase values are kept at zero. The HSLL achieved in a given scenario $\left(H S L L_{A}\right)$ is therefore different from the targeted HSLL $\left(H S L L_{T}\right)$. It has been found that $H S L L_{A}<H S L L_{T}, \forall H S S L_{T}$.

Fig 1 shows $H S L L_{A}$ for different $\mathrm{N}$ with Chebyshev and DPSS synthesis approaches. Based on datasheet, $K_{A}=5$ and $D=31 d B$ [2]. It is observed that $H S L L_{A}$ saturates after certain value of $H S L L_{T}$. It is also seen from Fig 1 that for $N=16$ (Chebyshev weights), there is sudden increase in $H S L L_{A}$ before saturation. The reason behind this anomaly is explained in Fig 2. The peaks are evaluated using findpeaks(.) function by MATLAB, which ignores side lobe adjacent to the main lobe if there is no null formed in between.

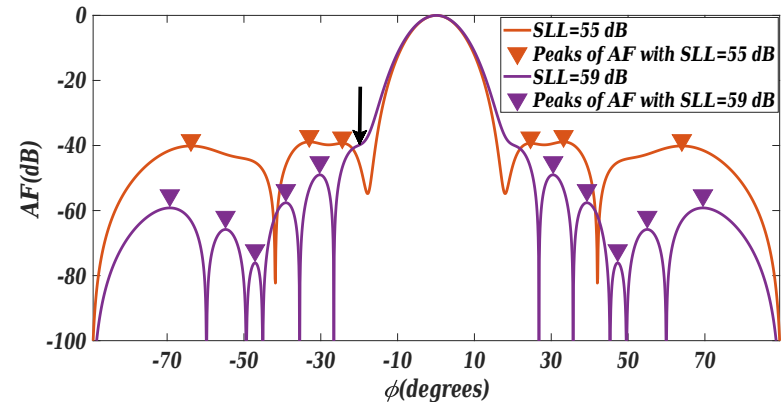

Fig. 2: Evaluation of peaks using findpeaks(.) function by MATLAB.

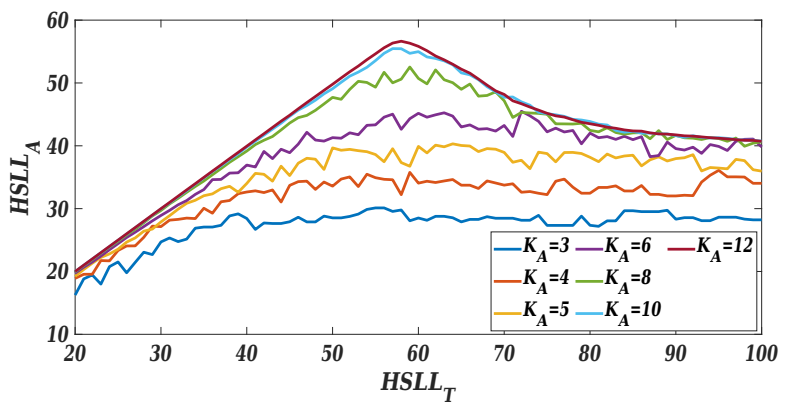

Fig. 3: Saturation of $H S L L_{A}$ for different values of $K_{A}$ for $N=64$.

This is shown for the case in which $H S L L_{T}=59 \mathrm{~dB}$. The black arrow points towards a premature null between the main and first left side lobe. Another plot in the same figure shows that when $H S L L_{T}=55 \mathrm{~dB}$, the side lobes adjacent to the main lobe are fully formed. The reason behind the incomplete null formations in the former case is due to the quantized amplitudes used as polynomial [13] coefficients while calculating $A F$.

The effect of $K_{A}$ is explored next. The variation of $H S L L_{A}$ with $H S L L_{T}$ for different $K_{A}$ values, while keeping the $D=31 d B$ is shown in Fig 3. As the number of bits are increased, the resolution of the attenuator increases which in turn increases $H S L L_{A}$. Thus, if higher value of $H S L L_{A}$ is desired then $K_{A}$ has to be increased.

Yet, even if the value $K_{A}$ is increased the $H S L L_{A}$ saturates after certain value of $H S L L_{T}$. This is because the attenuator will not be able to realize the amplitude values for the trailing elements as shown in Fig 4, for a Chebyshev array with $N=$ 64. It is clear that this worsens for DPSS array because this has higher suppression of farther side lobes than Chebyshev.

\section{B. Quantized Phase Shifters}

Let $\left\{\psi_{n}\right\}_{n=1}^{N}$ denote the phases in radians, i.e., $\psi_{n}=$ $n k d \sin \phi_{o}$. The resolution of the phase shifter is given by $\Delta_{p}=2 \pi / 2^{K_{p}}, K_{p}$ being the number of bits. Therefore, the quantized phases are given by

$$
\hat{\psi}_{n}=\Delta_{p}\left[\frac{\psi_{n}}{\Delta_{p}}\right]
$$




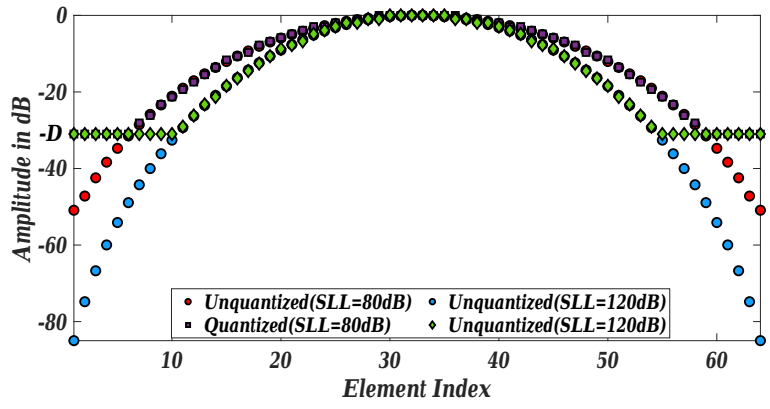

Fig. 4: Quantized and unquantized Chebyshev amplitudes with $D=31 d B$ and $K_{A}=5$.

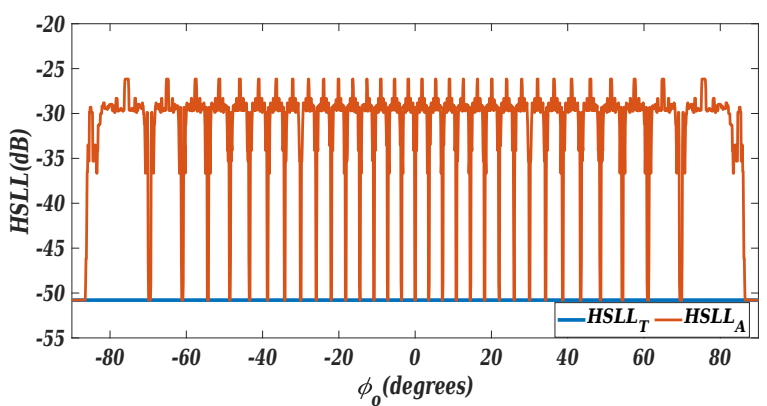

Fig. 5: Degradation in $H S L L$ when scanned over the useful space: $\Omega_{\phi}$ for $N=16, K_{p}=5$ and $H S L L_{T}=50.8 d B$.

Typically, phase of each element is rounded separately. This method causes negligible beam pointing error but does appear to have an impact on the HSLL.

Fig 5 shows the degradation of HSLL in the antenna array pattern when its main beam is scanned through different angles $\left(\phi_{o}\right)$. In this case, an array with DPSS amplitude weights for $N=16$ is used without quantization. It can be seen that there is no HSLL degradation for certain angles. These angles are obtained using

$$
\phi_{o, z}=\sin ^{-1}\left(\frac{n \Delta_{p}}{\pi}\right), n \leq 2^{K_{p}-1}
$$

At other angles, the HSLL is degraded. Angles at which the degradation is maximum $\left(H S L L_{A} \approx 26.1 \mathrm{~dB}\right.$ in this case) lie approximately in the middle of two adjacent $\phi_{o, z}$ values. The number of angles with maximum degradation is one less than the number of angles with no degradation $\left(\phi_{o, z}\right)$. Note that the values of $\phi_{o, z}$ are not periodic even though the quantization is uniform. It is also observed that the value of $H S L L_{A}$ lies between 26 and $30 d B$ for most scan angles, even when $H S L L_{T}=50 \mathrm{~dB}$ with $K_{p}=5$.

It may be of interest to investigate the array factors corresponding to some of these scan angles. The array factors corresponding to a value of $\phi_{o, z}$ and two other scan angles nearby, are plotted in Fig 6. The other patterns indicate no or partial formation of side lobes, due to quantized phases used.

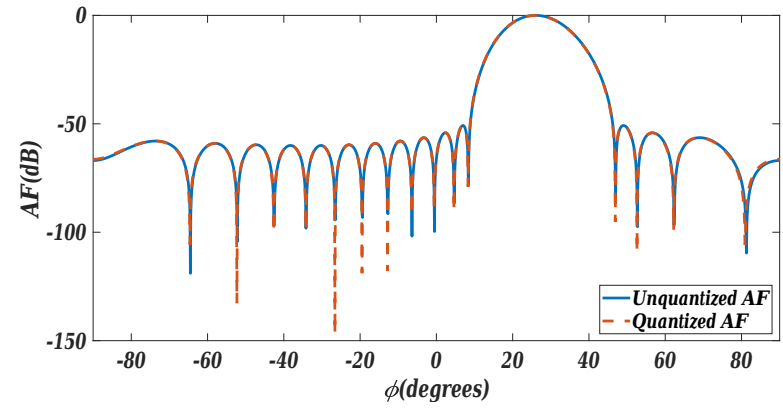

(a) $\phi_{o}=26^{\circ}$ (minimum degradation)

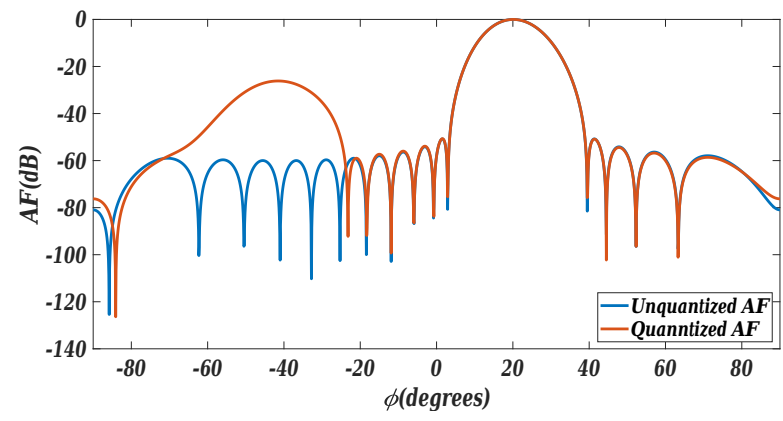

(b) $\phi_{o}=20.1^{\circ}$ (worst degradation)

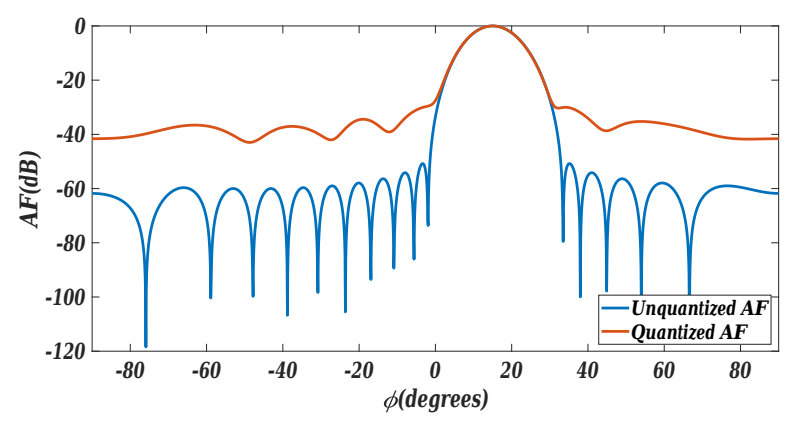

(c) $\phi_{o}=15^{\circ}$ (moderate degradation)

Fig. 6: Array Factor for $N=16$ with true DPSS weights and $K_{p}=5$ at different scan directions.

\section{AN APPROACH TO IMPROVE HSLL}

Scanning of main beam in a specified direction requires progressive phase shifts starting from the first antenna element to the Nth antenna element. But, quantizing the phase shifts according to (5) violates this basic requirement. The phase shifts at different antenna elements experience random quantization errors causing random SLL overshoots and hence degrading the HSLL. Therefore a new quantization method is proposed for phases.

First, the phase shift required at the first element to steer the main beam at $\phi_{o}$,

$$
\hat{\psi_{1}^{\prime}}=\Delta_{p}\left[\frac{\psi_{1}}{\Delta_{p}}\right]
$$

where, $\hat{\psi_{1}}{ }^{\prime}=\pi \sin \phi_{o}$. Phases of subsequent elements are 


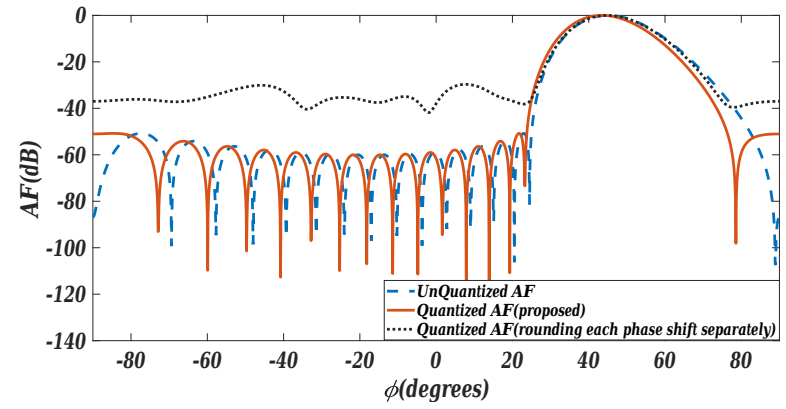

Fig. 7: Comparison of Array Factors with and without quantization of phases for $N=16$ and $\phi_{o}=45^{\circ}$. The proposed method achieves the $H S L L_{T}=50.8 d B\left(K_{p}=5\right)$.

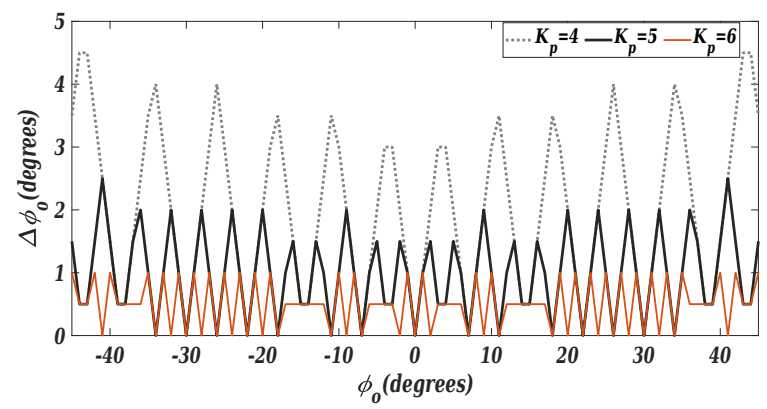

Fig. 8: Magnitude of Beam pointing error caused by progressive quantized phase shifting.

obtained from $\hat{\psi_{1}^{\prime}}$ by simple progressive multiplication,

$$
\hat{\psi_{n}^{\prime}}=n \hat{\psi_{1}^{\prime}}
$$

The generated quantized phases will be available in the beamformer's codebook. Therefore, the HSLL will not degrade due to phase quantization for all scan angles, when the proposed approach is used. Fig 7 compares the AF without phase quantization, AF obtained by quantizing each phase shift separately and finally the AF with phase shifts quantized using the proposed method for $N=16$ and $\phi_{o}=45^{\circ}$. This shows that the proposed method does not degrade HSLL even for the case where all the parasitic lobes appear to merge. This may be attributed to the fact the the proposed approach reinstates progressive phase shifts.

Although the proposed method improves HSLL requirements, it causes the beam to scan with discrete steps, causing the beam pointing errors to vary with scan angles. The quantized phase of the first element can be written as $\hat{\psi}_{1}=\psi_{1}+$ $\delta \psi_{1}$. This phase is multiplied by $n$ for $n^{\text {th }}$ antenna element. Thus, the phase at the $n^{t h}$ element is $\hat{\psi}_{n}=n \psi_{1}+n \delta \psi_{1}$. The additional progressive shift of $e_{n}=n \delta \psi_{1}$ across the ULA causes the beam to point in slightly different direction. For a greater number of bits $K_{p}$, the less is this error as depicted in Fig 8. With $K_{p}=5$, the beam pointing error in the scanning range $-45^{\circ} \leq 45^{\circ}$ is $\leq 2.5^{\circ}$ with an RMS value of $1.2^{\circ}$. Therefore, the proposed method can also be seen to directly provide $\phi_{o, z}$ beams discussed in Section IIB even when the intended scan direction is away from these angles. Since the proposed method provides only a limited number of possible beams for steering, it may cause signal attenuation at certain angles. This may affect the system dynamic range, and varies with other design parameters. Since the beam becomes narrower for larger arrays, the impact of this may become significant. Therefore, the proposed method is limited to small or moderately sized arrays such as those used in the mm wave $5 \mathrm{G}$ communications.

\section{CONCLUSIONS}

Performance of phased arrays using finite resolution control circuitry was analysed in detail. The limits on HSLL values that can be achieved using limited hardware were studied extensively. Dynamic range and resolution of attenuators limits the achieved HSLL. It has been found that the achieved HSLL range between 25 to $40 \mathrm{~dB}$ when $31 \mathrm{~dB}$ dynamic range is assumed. The achieved HSLL depends on the array size and number of control bits.

A similar degradation in HSLL is observed when phase shifters with a finite resolution are used with the conventional phase quantization approach. A new approach is proposed here to eliminate this HSLL degradation in moderately long arrays.

\section{REFERENCES}

[1] W. Hong, Z. H. Jiang, C. Yu, J. Zhou, P. Chen, Z. Yu, H. Zhang, B. Yang, X. Pang, M. Jiang, Y. Cheng, M. K. T. Al-Nuaimi, Y. Zhang, J. Chen, and S. He, "Multibeam antenna technologies for $5 \mathrm{~g}$ wireless communications," IEEE Transactions on Antennas and Propagation, vol. 65, no. 12, pp. 6231-6249, Dec 2017.

[2] $28 \mathrm{GHz}$ Silicon $5 G \mathrm{Tx} / \mathrm{Rx}$ Quad Core IC, Anokiwave mmW Solutions, 4 2018, rev. V11P.

[3] M. Smith and Y. Guo, "A comparison of methods for randomizing phase quantization errors in phased arrays," IEEE Transactions on Antennas and Propagation, vol. 31, no. 6, pp. 821-828, November 1983.

[4] S. Taheri and F. Farzaneh, "New methods of reducing the phase quantization error effects on beam pointing and parasitic side lobe level of the phased array antennas," in 2006 Asia-Pacific Microwave Conference, Dec 2006, pp. 2114-2117.

[5] U. K. Revankar, K. S. Beenamole, K. Sreenivasulu, and K. M. Veerabhadra, "Sidelobe minimisation in active phased arrays," IETE Technical Review, vol. 18, no. 2-3, pp. 191-196, 2001. [Online]. Available: https://doi.org/10.1080/02564602.2001.11416961

[6] W. P. M. N. Keizer, "Low sidelobe phased array pattern synthesis with compensation for errors due to quantized tapering," IEEE Transactions on Antennas and Propagation, vol. 59, no. 12, pp. 4520-4524, Dec 2011.

[7] V. Monga and K. J. Vinoy, "Equal sidelobe patterns for microstrip patch antenna arrays," in 2017 IEEE International Conference on Antenna Innovations Modern Technologies for Ground, Aircraft and Satellite Applications (iAIM), Nov 2017, pp. 1-5.

[8] K. J. Vinoy and M. A. Anilkumar, "Efficient synthesis of scanned conformal arrays with chebyschev patterns," in 2018 IEEE Indian Conference on Antennas and Propogation (InCAP), Dec 2018, pp. 1-4.

[9] C. A. Balanis, Antenna Theory: Analysis and Design. New York, NY, USA: Wiley-Interscience, 2005.

[10] D. Slepian and H. O. Pollak, "Prolate spheroidal wave functions, fourier analysis and uncertainty — i," The Bell System Technical Journal, vol. 40, no. 1, pp. 43-63, Jan 1961.

[11] S. Prasad, "On the index for array optimization and the discrete prolate spheroidal functions," IEEE Transactions on Antennas and Propagation, vol. 30, no. 5, pp. 1021-1023, Sep. 1982.

[12] T. Trump, "A robust adaptive sensor array with slepian sequences," in 2012 IEEE 7th Sensor Array and Multichannel Signal Processing Workshop (SAM), June 2012, pp. 121-124.

[13] S. A. Schelkunoff, "A mathematical theory of linear arrays," The Bell System Technical Journal, vol. 22, no. 1, pp. 80-107, Jan 1943. 